\title{
Magnetization of YBCO film with ac travelling magnetic waves of relatively short wavelengths
}

Wei Wang, and Tim Coombs

Citation: Appl. Phys. Lett. 110, 072601 (2017); doi: 10.1063/1.4976515

View online: https://doi.org/10.1063/1.4976515

View Table of Contents: http://aip.scitation.org/toc/apl/110/7

Published by the American Institute of Physics

\section{Articles you may be interested in}

Voltage induced mechanical/spin wave propagation over long distances

Applied Physics Letters 110, 072401 (2017); 10.1063/1.4975828

Giant interfacial perpendicular magnetic anisotropy in $\mathrm{MgO} / \mathrm{CoFe} / \mathrm{capping}$ layer structures

Applied Physics Letters 110, 072403 (2017); 10.1063/1.4976517

Mechanism of a high- $\mathrm{T}_{\mathrm{C}}$ superconducting flux pump: Using alternating magnetic field to trigger flux flow Applied Physics Letters 107, 142601 (2015); 10.1063/1.4932950

Voltage-ampere characteristics of YBCO coated conductor under inhomogeneous oscillating magnetic field Applied Physics Letters 108, 262601 (2016); 10.1063/1.4955266

Direct measurement of the vortex migration caused by traveling magnetic wave Applied Physics Letters 104, 032602 (2014); 10.1063/1.4862788

Room temperature insulating ferromagnetism induced by charge transfer in ultrathin (110) $\mathrm{La}_{0.7} \mathrm{Sr}_{0.3} \mathrm{MnO}_{3}$ films

Applied Physics Letters 110, 072405 (2017); 10.1063/1.4976699

\section{COMSOL} CONFERENCE 2018 BOSTON 


\title{
Magnetization of YBCO film with ac travelling magnetic waves of relatively short wavelengths
}

\author{
Wei Wang ${ }^{1, a)}$ and Tim Coombs ${ }^{2}$ \\ ${ }^{1}$ School of Electrical Engineering and Information, Sichuan University, Chengdu 610065, China \\ ${ }^{2}$ Electrical Engineering Division, Department of Engineering, University of Cambridge, Cambridge CB3 OFA, \\ United Kingdom
}

(Received 26 October 2016; accepted 31 January 2017; published online 14 February 2017)

\begin{abstract}
The magnetizations of the YBCO film with ac travelling magnetic waves of relatively short wavelengths were studied. The results have verified that the reported "intermediate value" of the superconducting current density [Wang et al., Appl. Phys. Lett. 104(3), 032602 (2014)] was caused by the existence of multiple transition regions in the sample: the magnetic poles induce $\pm J_{C}$ in the pole regions, which produces two transition regions within each wavelength $\lambda\left(+J_{C} \rightarrow-J_{C} \rightarrow+J_{C}\right.$, and vice versa, while the symbol $\rightarrow$ indicates the transition region). The current densities in the transition region are with intermediate values, which are smaller than the critical value. In case of relatively short wavelength, there are multiple transition regions, which occupy a large fraction of the YBCO sample with intermediate current values. Moreover, the wavelike current distributions might help explain the flux transportation and dc output voltage in HTS flux pump. Published by AIP Publishing. [http://dx.doi.org/10.1063/1.4976515]
\end{abstract}

AC travelling magnetic waves have positive and negative magnetic poles moving in space, they are clearly distinguished from homogeneous oscillating magnetic field, as the latter has a nil magnetic gradient. In large scale applications, such as rotational superconducting electric machines, the superconducting stator windings are subjected to ac travelling magnetic waves (rotor field) other than the homogeneous oscillating magnetic field. $\mathrm{Up}$ to date, the magnetization problems of second-generation $(2 \mathrm{G})$ high temperature superconducting (HTS) coated-conductor (CC) in ac travelling magnetic waves have not been clearly understood, due to limited literature on this subject. However, the ac magnetization losses caused by ac travelling magnetic waves is critical in the design of a superconducting electric machine. $^{2}$

The HTS flux pump technology ${ }^{3}$ faces the same magnetization problem, which has not been clearly answered at this stage. In the HTS flux pump, ac travelling magnetic wave was applied to a $2 \mathrm{G}$ HTS CC, while the dc output voltage was induced in the longitudinal direction. ${ }^{4}$ This $2 \mathrm{G}$ HTS CC which outputs dc voltage was named as the superconducting stator. The superconducting stator short-circuits an HTS coil with soldering to form a resistive close-loop. The induced dc output voltage charges the HTS coil, and the dc current was induced in the close-loop. The origination of the dc output voltage can hardly be explained by the conventional theories such as normal zone propogation, ${ }^{5}$ nor has been clearly supported by the classic magnetization model. ${ }^{6,7}$ The physics of HTS flux pump phenomenon is still under extensive discussions. ${ }^{8}$ Explanations are required from studying its unique magnetization behaviours and vortex dynamics.

To understand the above mentioned problem, a "circular-type magnetic flux pump" (CTMFP) device ${ }^{9-11}$

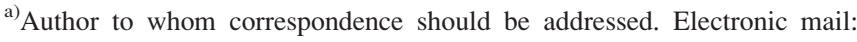
weiwangca283@gmail.com
}

was previously built, to study the magnetization of HTS $\mathrm{YBa}_{2} \mathrm{Cu}_{3} \mathrm{O}_{7-\delta}$ film in ac travelling magnetic waves. In the CTMFP experiments, both the YBCO film and the applied field are axial-symmetric, while the magnetization behaviour can be directly compared with the classic magnetization model for type-II superconductor. ${ }^{6,7}$ Previously, we have found that, the ac travelling wave compromises the preestablished critical magnetic gradient in the YBCO sample. A numerical model was built based on the finite-element method (FEM) with Maxwell's Equations and E-J power relationship, which accurately reproduced the magnetization process. The numerical model has shown that, the ac travelling magnetic waves have caused the superconducting current densities with "intermediate values," which were smaller than its critical value. ${ }^{1}$ In this paper, we have built an updated CTMFP device, shortening the produced wavelengths to half and quarter of the previous experiment. ${ }^{10}$ An updated numerical model have also been built to reveal the causation of the induced intermediate values of current densities.

Fig. 1(a) shows the two identical magnets of the updated CTMFP device, while Fig. 1(b) shows the structure. The two CTMFP magnets were installed co-axially, while a 2 in. diameter YBCO film was installed in the middle gap between the CTMFP magnets, sharing the same axis with the magnets. The geometry of the setup is axial-symmetric. The phase windings in the CTMFP magnets generate axialsymmetric ac travelling magnetic waves, ${ }^{10}$ while the flux are perpendicular to the a-b plane of the YBCO film. The detailed experimental setup can be found elsewhere. ${ }^{1,10}$ In the updated CTMFP magnets, the width of the teeth and slots of each phase winding is $1.25 \mathrm{~mm}$, which is one quarter of the original CTMFP magnets. ${ }^{10}$

In this work, we defined a parameter $\delta$ to describe the relative size of the YBCO sample to the wavelength

$$
\delta=r / \lambda,
$$


(a)

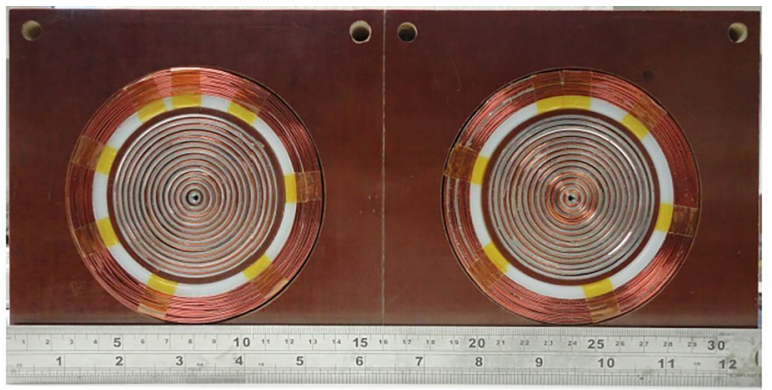

(b)

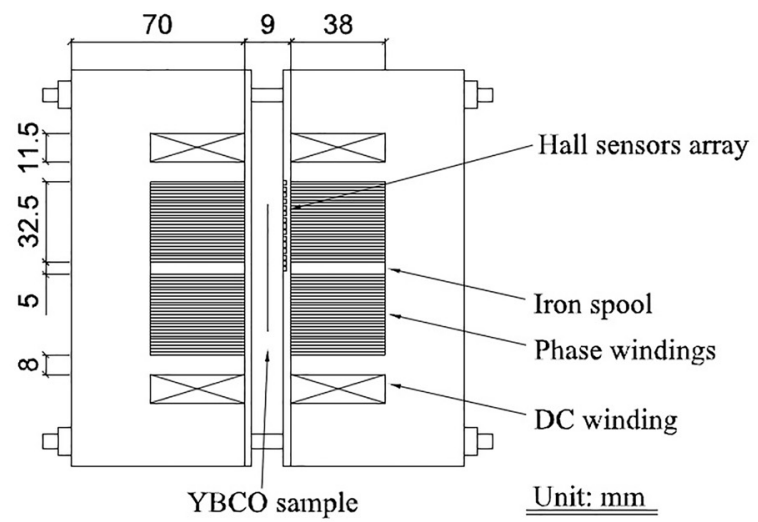

(c)

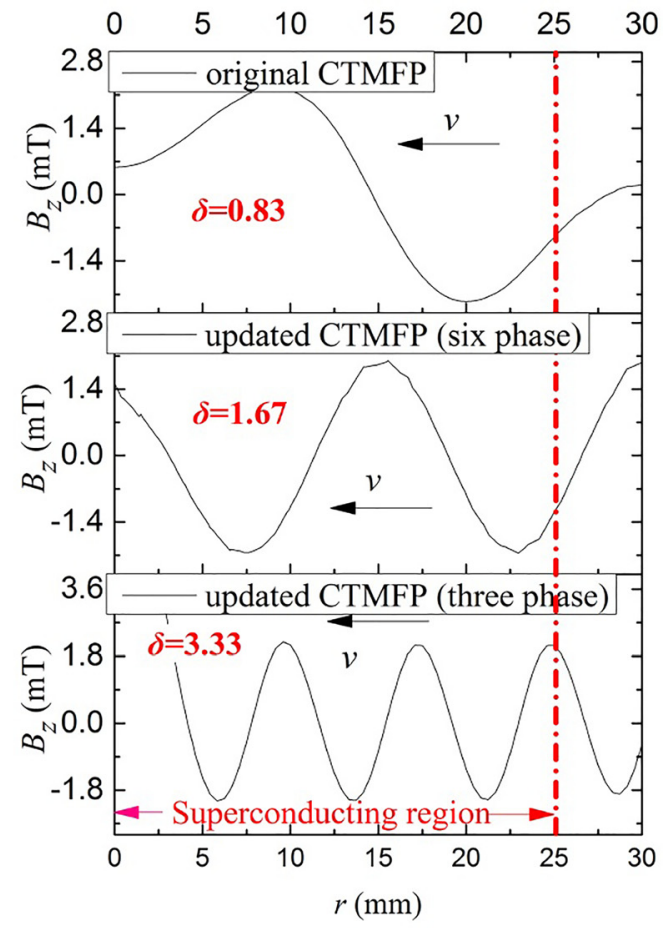

FIG. 1. (a) the two identical magnets of the updated CTMFP device, each contains axial-symmetric phase windings and dc winding, while the width of the teeth and slots of each phase winding is $1.25 \mathrm{~mm}$; (b) the structure of the updated CTMFP; (c) the waveforms produced by the original and updated CTMFP magnets, with three different wavelengths.

while $r=25 \mathrm{~mm}$ is the radius of the YBCO film in the experiment; $\lambda$ is the wavelength of the ac travelling magnetic wave produced by CTMFP magnets.

It is clear that, $\delta$ in Eq. (1) shows the number of magnetic pole-pairs in the superconducting region. Fig. 1(c) shows the waveforms produced by the original and updated CTMFP magnets, with three different wavelengths: (1) $\delta=0.83$ produced by original $\mathrm{CTMFP}^{10}$ ( $\lambda=30 \mathrm{~mm}$, single pole); (2) $\delta=1.67$ produced by updated CTMFP with a six phase connection ( $\lambda=15 \mathrm{~mm}$, three poles); (3) $\delta=3.33$ generated by updated CTMFP in three phase connection $(\lambda=7.5 \mathrm{~mm}$, six poles). For a given field amplitude, higher $\delta$ provides more moving poles in the superconducting sample region ( $r \leq 25 \mathrm{~mm}$ ), along with a stronger field inhomogeneity.

The 2 in. diameter $\mathrm{YBa}_{2} \mathrm{Cu}_{3} \mathrm{O}_{7-\delta}$ film was prepared by Ceraco Ceramic Coating GmbH, Germany. The thickness of the YBCO layer is $200 \mathrm{~nm}$, which was deposited on $\mathrm{Al}_{2} \mathrm{O}_{3}$ wafer. The sample was sealed in a Tufnol holder to protect it from humidity and scratch. The details of the YBCO film can be found elsewhere. ${ }^{10}$ The flux densities were measured with fifteen Hall sensors installed $1.5 \mathrm{~mm}$ above the sample surface as shown in Fig. 1(b). The Hall sensor spacing is $2.5 \mathrm{~mm}$. A multi-channel voltmeter Agilent U2353A was used to acquire the Hall voltages, which was calibrated and recorded with a Labview program.

The experiment procedure is: Step 1, the YBCO sample was zero-field cooled in liquid nitrogen in $77 \mathrm{~K}$; Step 2, the homogeneous dc-bias field $B_{d c}=11.5 \mathrm{mT}$ was applied to fully penetrate the sample, after $30 \mathrm{~s}$ of flux creep, then the magnetic flux densities were measured and recorded as "before travelling wave" (BTW); Step 3, the axial-symmetric ac travelling magnetic wave was applied for six cycles with the frequency $f=0.1 \mathrm{~Hz}(T=10.0 \mathrm{~s})$; Step 4, the ac travelling wave was turned off, and the magnetic flux densities were measured and recorded as "after travelling wave" (ATW).

An updated numerical model was built based on the FEM method with Maxwell's Equations and the $E-J$ power relationship, to simulate the complete magnetization process during the experiments. The governing equation is expressed as 1,11

$$
\mu_{r} \mu_{0} \frac{\partial \mathbf{H}}{\partial t}+\nabla \times(\rho \nabla \times \mathbf{H})=0
$$

while the magnetic field $\mathbf{H}=\left[H_{r}, H_{z}\right]^{\mathrm{T}}$, which is with cylindrical coordinates; the non-linear $E-J$ power law is used for the YBCO sample, which gives its resistivity as $\rho=J^{n-1} E_{C} /$ $J_{C}{ }^{n}$, with a critical current density $J_{C}=1.87 \mathrm{MA} / \mathrm{cm}^{2}$, which is comparable to a commercial $2 \mathrm{G}$ HTS CC; the index value $n=30$, the criterion $E_{C}=10^{-4} \mathrm{~V} / \mathrm{m}$. The field dependence of $J_{C}$ on magnetic flux density $\mathbf{B}$ is not considered in the model.

In both the experiment and simulation, it was found that the ac travelling wave was accompanied by a pulsating field (homogeneous oscillating field) as the inverter was switched on. This pulsating wave is undesired, which was caused by the "end effect" that is commonly found in linear electric machines, due to the incontinuity of the magnetic circuit. ${ }^{12}$ In the current experimental setup, this undesired pulsating field can hardly be eliminated, while the ac field generated by phase windings is expressed as

$$
\mathbf{B}_{a c}=\vec{e}_{z}\left\{B_{p u l s} \sin (\omega t)+B_{t r} \sin \left[\left(\frac{2 \pi}{\lambda}\right)(r-v t)\right]\right\},
$$

while $B_{\text {puls }}$ is the amplitude of the pulsating field, $\omega=2 \pi f$ is the angular speed, $f=0.1 \mathrm{~Hz}$ is the frequency; $B_{t r}$ is the 
amplitude of the ac travelling magnetic wave, $\lambda$ is the wavelength, $v=\lambda f$ is the travelling velocity.

In the numerical studies, the undesired pulsating field $B_{p u l s}$ can been eliminated, and a pure ac travelling wave will be applied, as will be discussed later.

The experimental and numerical results for $\delta=1.67$ $(\lambda=15.0 \mathrm{~mm})$ and $\delta=3.33(\lambda=7.5 \mathrm{~mm})$ are shown in Figs. 2(a) and 2(b), respectively. It is clear that the numerical model has accurately reproduced the experiments in all cases, even though the YBCO film was working in complicated field conditions as described in Eq. (3). The validity of the numerical model has been solidly verified by the experiments. The strategy of the following work is to use the verified numerical model to apply a pure ac travelling wave for the YBCO sample.

In the following numerical studies, the undesired pulsating field $B_{\text {puls }}$ was eliminated by imposing an anti-phase homogeneous oscillating field, and a pure ac travelling wave was applied. The homogeneous dc-bias field remains as $B_{d c}=11.5 \mathrm{mT}$, while the amplitudes of the ac travelling
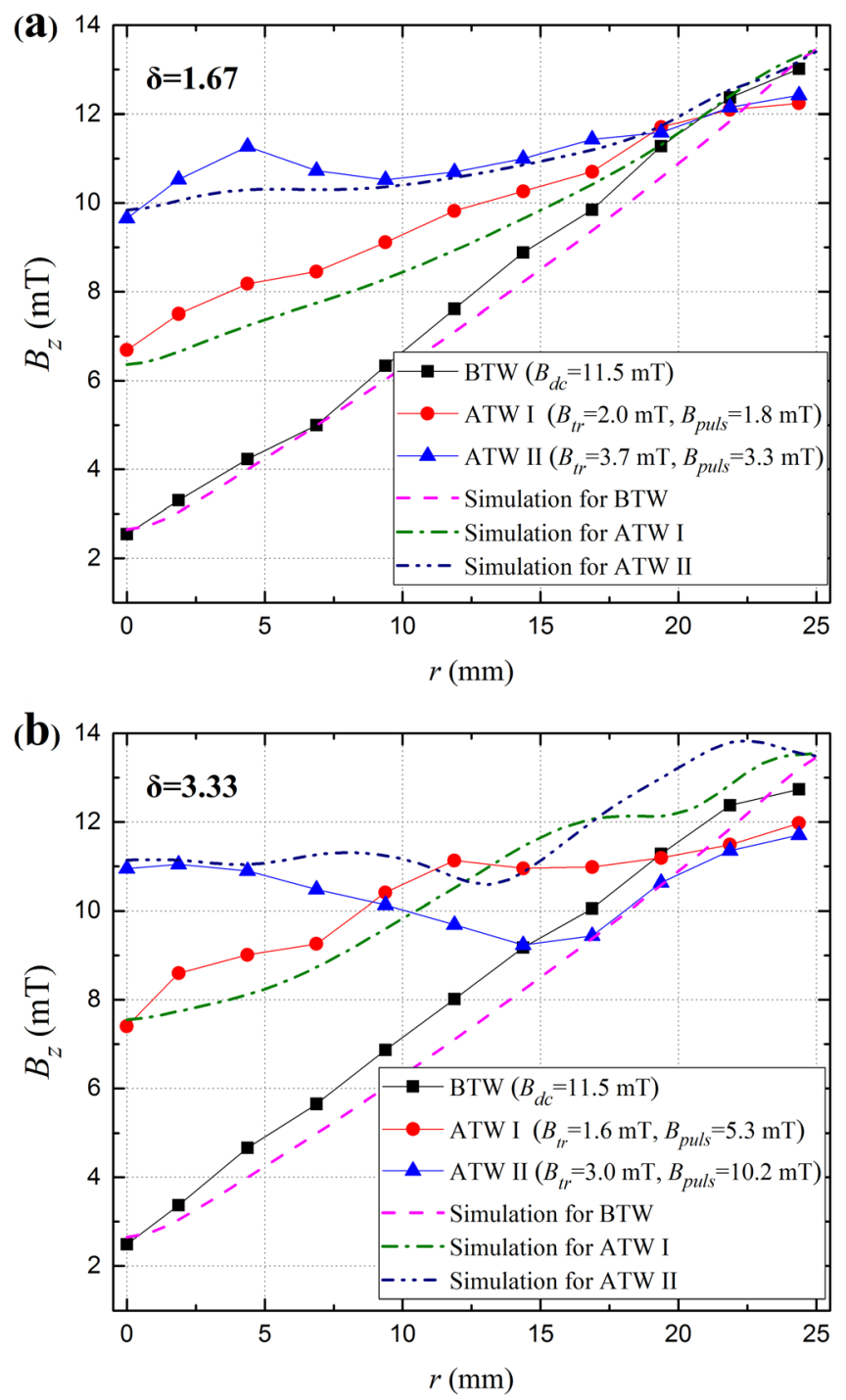

FIG. 2. The experimental and simulating results of the sample magnetized by the updated CTMFP in different wave conditions, the magnetic field was measured $1.5 \mathrm{~mm}$ above the sample surface (a) $\lambda=15.0 \mathrm{~mm}(\delta=1.67)$; (b) $\lambda=7.5 \mathrm{~mm}(\delta=3.33)$. waves were all set as $B_{t r}=2.0 \mathrm{mT}$ for three wavelength conditions $(\delta=0.83,1.67,3.33)$. The frequency remains as $f=0.1 \mathrm{~Hz}$. The magnetization procedure is the same as described in the experiment. The calculated ATW flux densities at $1.5 \mathrm{~mm}$ above the sample surface are shown in Fig. 3 (a). The corresponding normalised superconducting current density $J_{S C} / J_{C}$ inside the sample is shown in Fig. 3(b). As can be verified from the model, the current densities are uniform through the thickness of the YBCO layer, due to the fact that the sample is very thin.

As shown in Figs. 3(a) and 3(b), after the dc-bias field was applied, the induced superconducting current density is about $-0.8 J_{C}$ cross the radius, which is about $20 \%$ smaller than its critical current density $J_{C}$, this is due to the relatively small $n$-value in the $E-J$ power relationship at $77 \mathrm{~K}$, which causes a clear flux creep after applying the dc field, and remains stable at about $-0.8 J_{C}$.

Fig. 3(a) shows a clear degradation of pre-established magnetic gradients after applying the ac travelling waves, similar to the results in the previous CTMFP experiment. ${ }^{1}$ That is to say, the ac travelling waves tend to flatten out the pre-established critical magnetic gradient in the YBCO film, making the value of magnetic gradient smaller than the critical value. The magnetic gradient degrades further with the decrease of wavelength $\lambda$ from $30 \mathrm{~mm}, 15 \mathrm{~mm}$ to $7.5 \mathrm{~mm}(\delta$ increases from $0.83,1.67$ to 3.33 ). The flux density at $r=0 \mathrm{~mm}$ reaches the highest value when $\delta=3.33$. Fig. 3(b) shows the corresponding current density distributions, which drastically decreased from $-0.8 J_{C}$ to varied intermediate values between $-0.6 J_{C}$ and $0.6 J_{C}$.

To understand why the intermediate current values appear in the YBCO film, it should be acknowledged that this magnetization problem is non-standard, which can hardly be explained by the conventional magnetization model based on homogeneous field conditions. The classic Bean's model ${ }^{6}$ has defined that the current density has only three possible values in type-II superconductors with a strong pinning (index value $n \rightarrow \infty): 0, \pm J_{C}$. The popular interpretations of the Bean's model have presumed the field condition as $\lambda \rightarrow \infty$ or $\delta=0$, i.e., the wavelength is much larger than the sample size, thereby the field is homogeneous in the sample region. ${ }^{7}$ In the Bean's limit $(\lambda \rightarrow \infty, \delta=0, n \rightarrow \infty)$, when a positive pole arrives at the sample region, it induces $-J_{C}$ cross the whole sample (similar to the curve "BTW" in Fig. 3(b)). Likewise, when a negative pole arrives, it induces $+J_{C}$ cross the whole sample. However, when the field is between positive and negative poles, there exists a "transition region" in which the current reverses direction from $-J_{C}$ to $+J_{C}$, and vice versa. The transition region travels with the applied field and disappears as the next pole has arrived. The current densities in the transition region are with intermediate values. However, in the Bean's model, the transition from $-J_{C}$ to $+J_{C}$ is immediate, because the large index value $n \rightarrow \infty$ enables a sharp transition, thus the length of the transition region is zero. Therefore, the intermediate values are considered to be non-existing in the hard type-II superconductor based on the Bean's limit.

In our experiments, there are multiple moving magnetic poles within the sample region, because the wavelengths are smaller than the sample size $(\delta>1)$. As discussed above, 


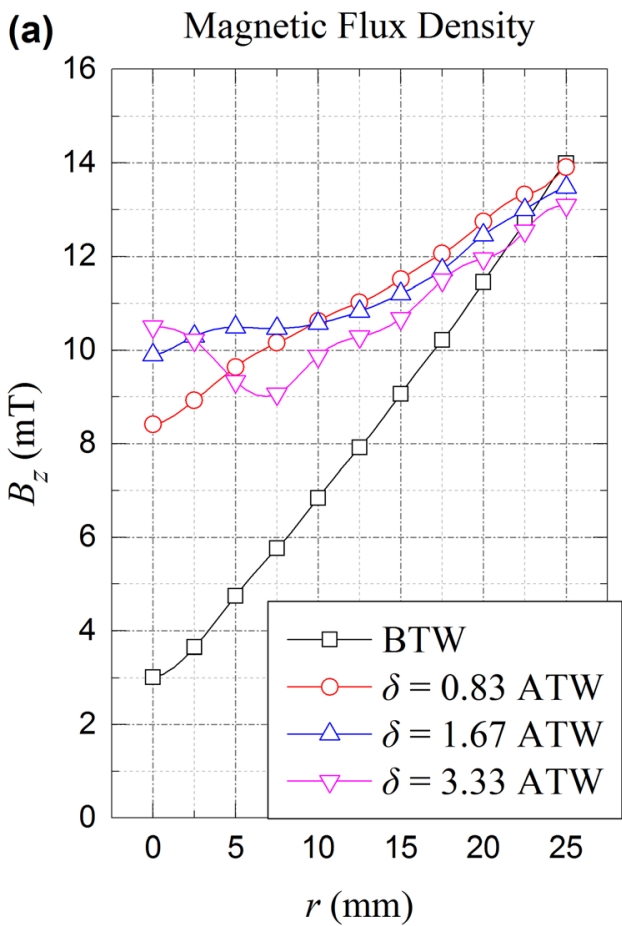

(b) Normalized SC current density

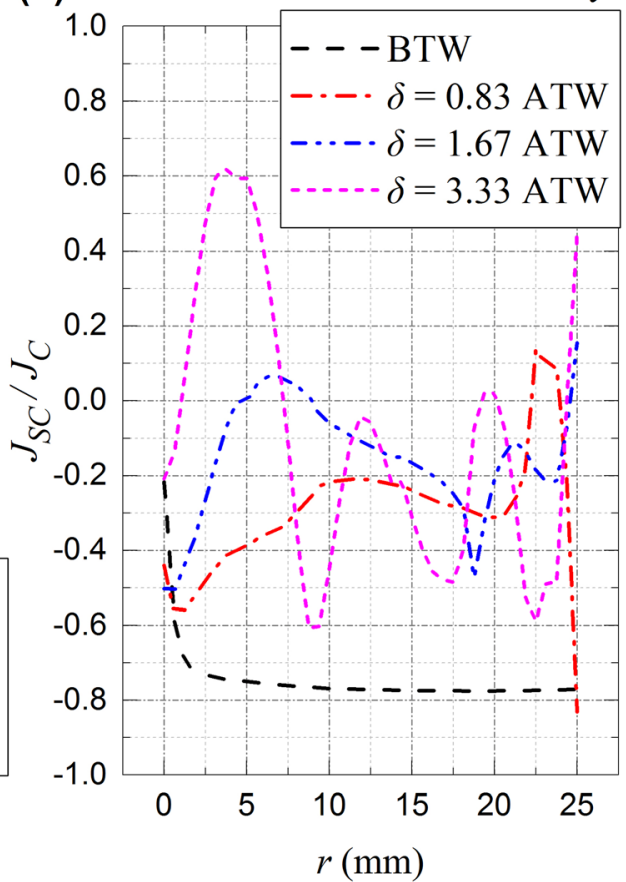

FIG. 3. The simulated results after applying a pure ac travelling wave. The homogeneous dc-bias field was set as $B_{d c}=11.5 \mathrm{mT}$; the amplitudes of the ac travelling waves were all set as $B_{t r}=2.0 \mathrm{mT}$ for three wavelength conditions $\lambda=30 \mathrm{~mm}, 15 \mathrm{~mm}$ to $7.5 \mathrm{~mm}$ $(\delta=0.83,1.67,3.33)$. The magnetization procedure is the same as in the experiment. (a) the simulated flux densities $1.5 \mathrm{~mm}$ above the sample surface before (BTW) and after (ATW) the ac travelling waves; (b) the simulated superconducting current densities cross the sample radius before (BTW) and after (ATW) the ac travelling waves. the magnetic poles induce $\pm J_{C}$ in the pole regions, which produces two transition regions within each wavelength $\lambda$ $\left(+J_{C} \rightarrow-J_{C} \rightarrow+J_{C}\right.$, and vice versa, while the symbol $\rightarrow$ indicates the transition region). In our study, as the index value of the YBCO film $(n=30)$ does not meet the Bean's limit $(n \rightarrow \infty)$, the length of the transition region is nonzero. Suppose the ith transition region has a length $\varepsilon_{i}$, which gives a total fraction $\sum_{i=1}^{[2 \delta]} \varepsilon_{i} / r$ of the YBCO film with intermediate values, which, causes the degradation of the preestablished critical magnetic gradient as shown in Fig. 3(a).

To verify above argument, we picked up a random moment during applying ac travelling waves, to show the transient current distributions. As shown in Figs. 4(a) and $4(\mathrm{~b})$, in case of $\lambda=15 \mathrm{~mm}(\delta=1.67)$ and $\lambda=7.5 \mathrm{~mm}$ $(\delta=3.33)$, the induced current densities are clearly periodical and wavelike. The induced current densities in the pole regions reach $\pm 0.8 J_{C}$. In the case $\lambda=15 \mathrm{~mm}(\delta=1.67)$, as marked in Fig. 4(a), the "wavelength" of the wavelike current distribution is $15.0 \mathrm{~mm}$, which equals to the applied wavelength; likewise, in the case $\lambda=7.5 \mathrm{~mm}(\delta=3.33)$, as marked in Fig. 4(b), the "wavelength" of the wavelike current distribution is $7.5 \mathrm{~mm}$, which equals to the applied wavelength.

Figs. 4(a) and 4(b) also verify that, there are two transition regions within each wavelength. The length of each transition region is non-zero, while the current densities are with the intermediate values. In Fig. 4(a), for $\delta=1.67$, there are clearly three transition regions, with a total fraction (a)

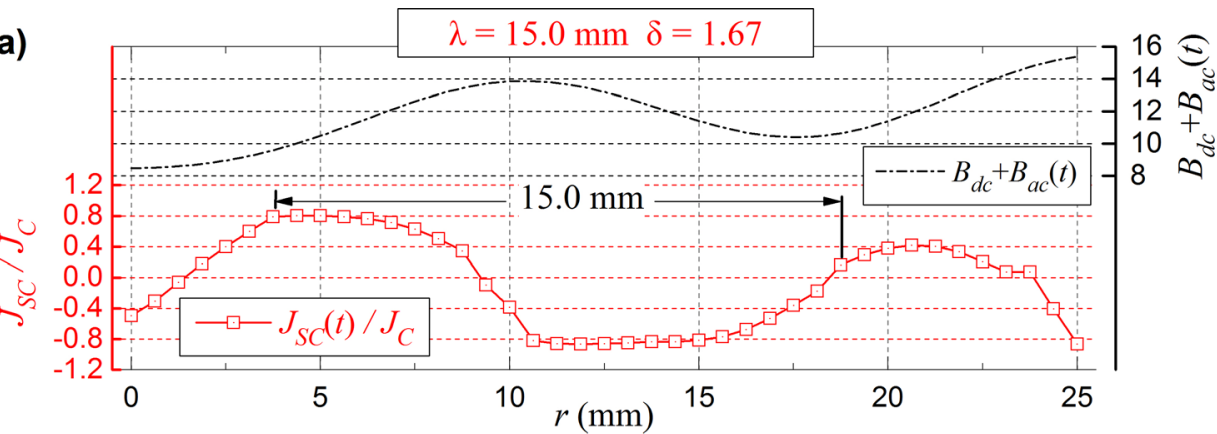

(b)

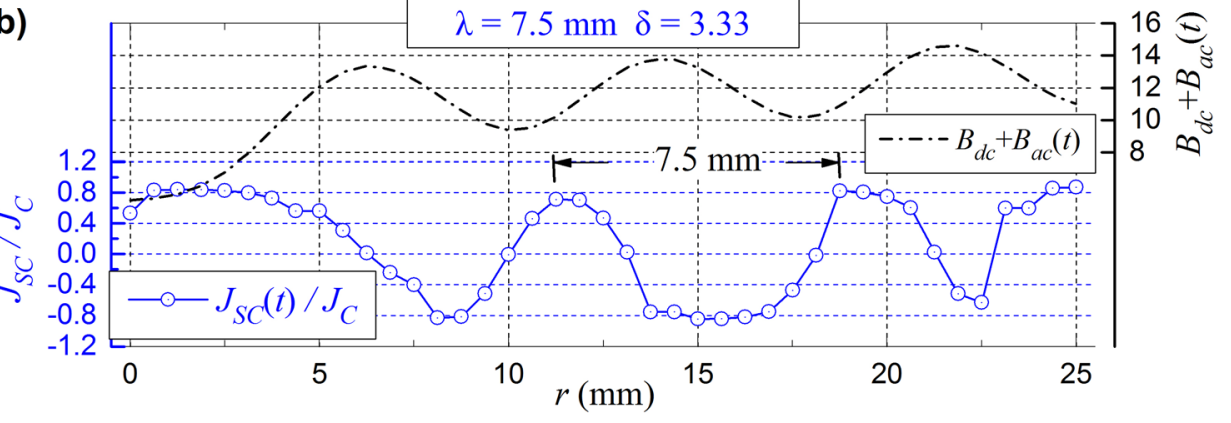

FIG. 4. Transient moments when the ac travelling waves were applied to the YBCO film. The waveforms are shown at the upper diagram, while the induced superconducting current densities are shown at the lower diagram. (a) $\lambda=15.0 \mathrm{~mm}(\delta=1.67)$; (b) $\lambda=$ $7.5 \mathrm{~mm}(\delta=3.33)$. 
$\sum_{i=1}^{3} \varepsilon_{i} / r$ of the YBCO sample with intermediate values, which occupies a relatively small fraction of the sample. However, in Fig. 4(b), for $\delta=3.33$, there are clearly six transition regions, with a total fraction $\sum_{i=1}^{6} \varepsilon_{i} / r$ of the YBCO sample with intermediate values, which occupies a large fraction of the YBCO sample. Meanwhile, the wavelike current distributions also lead to a smaller average current density compared to the critical value. Both the transition regions and the wavelike current distributions have caused the degradation of pre-established critical magnetic gradient as described in the experiments. The wavelike current distributions also suggest that, the short wavelength ac travelling wave might help transport the magnetic flux cross the YBCO film, which might help explain the dc output voltage in the HTS flux pump. However, this requires a further examination.

In this paper, we studied the magnetizations of a 2 in. diameter YBCO film with ac travelling magnetic waves of relatively short wavelengths, with updated CTMFP magnets and FEM model based on Maxwell's Equations and $E-J$ power relationship. The results have verified that, the reported "intermediate value" of the superconducting current density ${ }^{1}$ was caused by the existence of multiple transition regions in the sample: the magnetic poles induce $\pm J_{C}$ in the pole regions, while the two transition regions exist within each wavelength $\lambda\left(+J_{C} \rightarrow-J_{C} \rightarrow+J_{C}\right.$, and vice versa, while the symbol $\rightarrow$ indicates the transition region). The current densities in the transition region are with intermediate values, which are smaller than the critical value. In case of a relatively short wavelength, there are multiple transition regions, which cause a large fraction of the YBCO sample with intermediate current values. Meanwhile, the wavelike current distributions also lead to a smaller average current density compared with the critical value. Both the transition regions and the wavelike current distributions have caused the degradation of pre-established critical magnetic gradient. The wavelike current distributions also suggest that the short wavelength ac travelling waves might help transport the magnetic flux in the YBCO sample, which explains the dc output voltage in HTS flux pump. However, this requires further examination.
The author would like to acknowledge the helpful discussion with Professor Archie Campbell of the University of Cambridge. This work was supported by the National Natural Science Foundation of China under Grant No. 51607117.

${ }^{1}$ W. Wang, F. Spaven, M. Zhang, M. Baghdadi, and T. Coombs, Appl. Phys. Lett. 104(3), 032602 (2014).

${ }^{2}$ M. Zhang, M. Chudy, W. Wang, Y. Chen, Z. Huang, Z. Zhong, W. Yuan, J. Kvitkovic, S. V. Pamidi, and T. A. Coombs, IEEE Trans. Appl. Supercond. 23(3), 5900604 (2013); P. Song, T. M. Qu, L. F. Lai, M. S. Wu, X. Y. Yu, and Z. Han, Supercond. Sci. Technol. 29(5), 054007 (2016); Z. Min, Y. Weijia, K. Jozef, and P. Sastry, Supercond. Sci. Technol. 28(11), 115011 (2015); P. Enric and G. Francesco, Supercond. Sci. Technol. 25(1), 014008 (2012); F. Gömöry, M. Vojenčiak, E. Pardo, M. Solovyov, and J. Šouc, Supercond. Sci. Technol. 23(3), 034012 (2010); Q. Loïc, M. R. Zermeño Víctor, and G. Francesco, Supercond. Sci. Technol. 29(2), 024007 (2016); N. Amemiya, K. Miyamoto, S.-I. Murasawa, H. Mukai, and K. Ohmatsu, Phys. C: Supercond. 310(1-4), 30 (1998).

${ }^{3}$ C. Hoffmann, D. Pooke, and A. D. Caplin, IEEE Trans. Appl. Supercond. 21(3), 1628 (2011); R. M. Walsh, R. Slade, D. Pooke, and C. Hoffmann, IEEE Trans. Appl. Supercond. 24(3), 1 (2014); F. Lin, M. Koichi, L. Thibault, I. Yukikazu, and C. Tim, Supercond. Sci. Technol. 29(4), 04LT01 (2016); Z. Bai, G. Yan, C. Wu, S. Ding, and C. Chen, Cryogenics 50(10), 688 (2010).

${ }^{4}$ C. W. Bumby, Z. Jiang, J. G. Storey, A. E. Pantoja, and R. A. Badcock, Appl. Phys. Lett. 108(12), 122601 (2016).

${ }^{5}$ L. J. M. van de Klundert and H. H. J. ten Kate, Cryogenics 21(4), 195 (1981).

${ }^{6}$ C. P. Bean, Rev. Mod. Phys. 36(1), 31 (1964).

${ }^{7}$ W. J. Carr, AC Loss and Macroscopic Theory of Superconductors (Taylor \& Francis, London, 2001).

${ }^{8}$ J. Geng, K. Matsuda, L. Fu, J. F. Fagnard, H. Zhang, X. Zhang, B. Shen, Q. Dong, M. Baghdadi, and T. A. Coombs, J. Phys. D: Appl. Phys. 49(11), 11LT01 (2016); G. Jianzhao, K. Matsuda, F. Lin, S. Boyang, Z. Xiuchang, and T. A. Coombs, Supercond. Sci. Technol. 29(3), 035015 (2016); Z. Jiang, K. Hamilton, N. Amemiya, R. A. Badcock, and C. W. Bumby, Appl. Phys. Lett. 105(11), 112601 (2014).

${ }^{9}$ W. Wang, M. Zhang, C. Hsu, and T. Coombs, IEEE Trans. Appl. Supercond. 22(3), 5201304 (2012); W. Wang, M. Zhang, Z. Huang, Y. Zhai, Z. Zhong, F. Spaven, M. Baghdadi, and T. Coombs, IEEE Trans. Appl. Supercond. 24(3), 4600304 (2014); W. Wang, R. Semerad, F. Spaven, M. Zhang, C. H. Hsu, Z. Zhong, Y. Chen, Z. Huang, and T. A. Coombs, IEEE Trans. Appl. Supercond. 23(3), 8201104 (2013).

${ }^{10}$ W. Wang and T. A. Coombs, J. Appl. Phys. 113(21), 213906 (2013).

${ }^{11} \mathrm{~W}$. Wang and T. Coombs, Supercond. Sci. Technol. 28(5), 055003 (2015).

${ }^{12}$ O. Danielsson and M. Leijon, IEEE Trans. Magn. 43(7), 3197 (2007). 